\title{
EIGENVALUES OF LAPLACIANS ON A CLOSED RIEMANNIAN MANIFOLD AND ITS NETS
}

\author{
KOJI FUJIWARA \\ (Communicated by Peter Li) \\ Dedicated to Professor Hideki Ozeki on his 60th birthday
}

\begin{abstract}
We show that the eigenvalues of the Laplacian of a closed manifold $M$ is approximated in a certain sense by the eigenvalues of the Laplacian of the graph of a $\frac{1}{n}$-net in $M$ as $n \rightarrow \infty$. Our approximation needs no assumption on $M$ except for dimension.
\end{abstract}

\section{INTRODUCTION AND STATEMENT OF THE MAIN THEOREM}

In this paper, we study the relationship between the eigenvalues of the Laplacian of a closed Riemannian manifold and those of its net, a graph which approximates the manifold.

To recall the definition of the Laplacian on a graph $[B],[F]$, let $\Gamma$ be a connected finite graph, $V(\Gamma)$ the set of its vertices, and $E(\Gamma)$ the set of its directed edges. We assume there are no edges joining a vertex with itself and if two distinct vertices $x$ and $y$ are joined by an edge, which we denote $x \sim y$, then there are exactly two edges of opposite directions between them. We denote the edge from $x$ to $y$, if it exists, by $[x, y]$ or $-[y, x]$.

Length function $l: E(\Gamma) \rightarrow \mathbf{R}_{+}$is a positive function on $E(\Gamma)$ with $l([x, y])$ $=l([y, x])$. We define a weight function $m_{l}$ on $V(\Gamma)$ by

$$
m_{l}(x)=\sum_{x \sim y} l([x, y])
$$

where $\sum_{x \sim y}$ means to take the sum over all the vertices $y$ connected to $x$, and we sometimes write $m$ instead of $m_{l}$ for simplicity. We put

$$
\begin{aligned}
& L^{2}(V(\Gamma))=\{f: V(\Gamma) \rightarrow \mathbf{R}\}, \\
& L^{2}(E(\Gamma))=\{\phi: E(\Gamma) \rightarrow \mathbf{R} \mid \phi(-e)=-\phi(e)\},
\end{aligned}
$$

and define inner products for $f, g \in L^{2}(V)$ and $\phi, \psi \in L^{2}(E)$ by

$$
(f, g)=\sum_{x \in V(\Gamma)} m(x) f(x) g(x), \quad(\phi, \psi)=\frac{1}{2} \sum_{e \in E(\Gamma)} l(e) \phi(e) \psi(e) .
$$

Received by the editors December 1, 1993.

1991 Mathematics Subject Classification. Primary 58G25; Secondary 39B42.

The author was supported in part by Yukawa Postdoctral Fellowship of Osaka University. 
As an analogue of the exterior derivative, we define an operator $d: L^{2}(V) \longrightarrow$ $L^{2}(E)$ by

$$
d f([x, y])=\frac{f(x)-f(y)}{l([x, y])} \text { for } f \in L^{2}(V) .
$$

The adjoint operator $\delta: L^{2}(E) \longrightarrow L^{2}(V)$ is then given by

$$
\delta \phi(x)=\frac{1}{m(x)} \sum_{x \sim y} \phi([x, y]) \text { for } \phi \in L^{2}(E) .
$$

We define the Laplacian on $(\Gamma, l)$ by

$$
\Delta f(x)=\delta d f(x)
$$

Then we obtain $(\Delta f, f)=(d f, d f)$, and we can rewrite

$$
\Delta f(x)=\frac{1}{m(x)} \sum_{x \sim y} \frac{f(x)-f(y)}{l([x, y])} .
$$

The smallest eigenvalue $\lambda_{0}(\Gamma, l)$ for $\Delta$ is always 0 and the one-dimensional eigenspace for 0 consists of the constant functions, since $\Gamma$ is connected. We denote the $k$-th positive eigenvalue of $\Delta$ by $\lambda_{k}(\Gamma, l)$ :

$$
0=\lambda_{0}<\lambda_{1} \leq \lambda_{2} \leq \cdots \leq \lambda_{n} \text {, where } n=\sharp V-1 .
$$

Before discussing the general case for approximating the eigenvalues of the Laplacian on a closed Riemannian manifold by graphs, we give a simple example. Let $S^{1}$ be the unit circle and $\lambda_{k}\left(S^{1}\right)$ denote the $k$-th eigenvalue of the Laplacian on $S^{1}$. It is known that

$$
\left\{\lambda_{k}\left(S^{1}\right)\right\}_{k=1}^{\infty}=\{0, \underbrace{1,2^{2}, 3^{2}, 4^{2}, \ldots}_{\text {mult. }=2}\} .
$$

Let $\left(C_{n}, l_{n}\right)$ be a circle graph of $n$-vertices with length function $l_{n} \equiv 2 \pi / n$. We may directly calculate the values for $\lambda_{k}\left(C_{n}, l_{n}\right)$, which we denote by $\operatorname{spec}\left(C_{n}\right)$. If $n$ is odd,

$$
\operatorname{spec}\left(C_{n}\right)=\left(\frac{n}{2 \pi}\right)^{2} \times\{0, \underbrace{2\left(1-\cos \frac{2 \pi}{n}\right), \ldots, 2\left(1-\cos \frac{n-1}{n} \pi\right)}_{\text {mult. }=2}\} .
$$

If $n$ is even,

$$
\operatorname{spec}\left(C_{n}\right)=\left(\frac{n}{2 \pi}\right)^{2} \times\{0, \underbrace{2\left(1-\cos \frac{2 \pi}{n}\right), \ldots, 2\left(1-\cos \frac{n-2}{n} \pi\right)}_{\text {mult. }=2}, 4\} .
$$

Since $\lim _{n}\left(\frac{n}{2 \pi}\right)^{2} 2\left(1-\cos \frac{2 k}{n} \pi\right)=k^{2}$, we have

$$
\lim _{n \rightarrow \infty} \lambda_{k}\left(C_{n}\right)=\lambda_{k}\left(S^{1}\right) \quad \text { for each } k .
$$

To approximate the eigenvalues of the Laplacians on a closed Riemannian manifold $M$, we take an $\varepsilon$-net in $M$, which is a graph obtained in the fol- 
lowing way for $\varepsilon>0$. A subset of $M$ is called $\varepsilon$-separated if $d_{M}(x, y) \geq \varepsilon$ for distinct points $x, y$ of the set. Take a maximal (with respect to inclusion of sets) $\varepsilon$-separated subset $V$ in $M$, and join distinct points $x$ and $y$ of $V$ by two directed edges from $x$ to $y$ and from $y$ to $x$ if and only if $d_{M}(x, y) \leq 3 \varepsilon$. The resulting graph is termed an $\varepsilon$-net in $M$. It is known that a maximal $\varepsilon$-separated set exists for any $\varepsilon>0$ and the graph obtained from it is always connected $[\mathrm{K}]$. It is clear from the construction that an $\varepsilon$-net in $M$ approximates the manifold $M$ as a metric space. Moreover, it approximates the eigenvalues of Laplacian, which we state in the following theorem.

Theorem. Let $M^{d}$ be a d-dimensional closed Riemannian manifold and $\left(\Gamma_{n}, l_{n}\right)$ a $\frac{1}{n}$-net in $M$ with length function $l_{n} \equiv 1 / n$ for each $n \in \mathbf{N}$. Then

$$
\frac{1}{C} \limsup _{n \rightarrow \infty} \lambda_{k}\left(\Gamma_{n}, l_{n}\right) \leq \lambda_{k}(M) \leq C \liminf _{n \rightarrow \infty} \lambda_{k}\left(\Gamma_{n}, l_{n}\right)
$$

for each $k$, where $\lambda_{k}(M)$ is the $k$-th eigenvalue of the Laplacian on $M$ and $C$ is a number depending only on the dimension of the manifold, which satisfies $C \leq 2 \cdot 50^{d}$.

To show the inequalities in the Theorem, we do not need any assumptions on $M$ except for dimension, and the inequalities hold for any sequence of $\frac{1}{n}$ nets. But the rate of the convergence depends on the curvature of $M$. We will discuss this in the next section before giving the proof, since it would be needed for application.

At present, the constant $C$ depends exponentially on the dimension. However, from this theorem, we can know the rough behavior of the eigenvalues of the Laplacian on $M$ from that of $\Gamma_{n}$, which is easier to compute since the function space over $\Gamma_{n}$ has finite dimension for each $n$. We may expect sharper estimates for the eigenvalues taking a nice sequence of graphs, but the author does not know, for example, if the inequalities in the Theorem hold for a constant $C^{\prime}$ independent of the dimension for a suitably nice sequence of graphs for $M$. But it seems that we can choose a sequence of nets of a manifold whose eigenvalues do not converge to the ones of the manifold.

There have been many results on Laplacians on graphs using different definitions. For example, Dodziuk [D] studied a certain combinatorial Laplacian which carries more geometric information of the manifold than ours.

The author thanks Professor Steven Rosenberg, who brought him to this problem and gave valuable suggestion. He appreciates the comments of the referee on the proof.

\section{Proof of The TheOREM}

To prove the Theorem, we will need the following Lemma (see [B] and Chapter 1 of $[\mathrm{C}]$ ) called the minimax principle. In this section, we write $\Gamma$ instead of $V(\Gamma)$ for simplicity, and accordingly denote $L^{2}(V(\Gamma))$ by $L^{2}(\Gamma)$.

Lemma.

$$
\lambda_{k}(\Gamma)\left(\operatorname{resp} . \lambda_{k}(M)\right)=\inf _{\mathscr{F}_{k+1}} \sup _{f \in \mathscr{F}} \frac{(d f, d f)}{(f, f)}
$$

where $\mathscr{F}_{k+1}$ runs over linear subspaces of $L^{2}(\Gamma)$ (resp. $L^{2}(M)$ ) of dimension $k+1$. 
The expression $(d f, d f) /(f, f)$ is called the Rayleigh quotient of $f$.

The proof consists of two parts. First, to show $\lambda_{k}(M) \leq C \liminf _{n} \lambda_{k}\left(\Gamma_{n}\right)$, we construct a linear operator for each $n$

$$
S_{n}: L^{2}\left(\Gamma_{n}\right) \rightarrow C^{\infty}(M)
$$

which satisfies

$$
\frac{\left(d S_{n}(f), d S_{n}(f)\right)_{M}}{\left(S_{n}(f), S_{n}(f)\right)_{M}} \leq C \frac{(d f, d f)_{\Gamma_{n}}}{(f, f)_{\Gamma_{n}}},
$$

for sufficiently large $n$. Next, to show $\lim \sup _{n} \lambda_{k}\left(\Gamma_{n}\right) \leq C \lambda_{k}(M)$, we construct a linear operator

$$
T_{n}: C^{\infty}(M) \rightarrow L^{2}\left(\Gamma_{n}\right)
$$

for each $n$ with the following property. Let $\mathscr{F}$ be a finite-dimensional linear subspace of $C^{\infty}(M)$, and $\mathscr{F}(1)$ denote the subset $\{f \in \mathscr{F} \mid(f, f)=1\}$. Then for any $\varepsilon>0$, taking sufficiently large $n$, we have

$$
\frac{\left(d T_{n}(f), d T_{n}(f)\right)_{\Gamma_{n}}}{\left(T_{n}(f), T_{n}(f)\right)_{\Gamma_{n}}} \leq C \frac{(d f, d f)_{M}+\varepsilon}{(f, f)_{M}-\varepsilon},
$$

for each $f \in \mathscr{F}(1)$. From the above two estimates of the Rayleigh quotient, applying the Lemma, we can obtain the inequalities in the Theorem.

Constants. Here we list up in advance several geometric constants which we will use in the proof. For a point $x \in M$, we write the set $\{y \in M \mid d(x, y)<r\}$ by $B(x, r)$ and denote its volume in $M$ by vol $B(x, r)$. There exist positive constants $C_{1}, C_{2}, \ldots, C_{8}$ which depend only on the dimension of the manifold $d$ and satisfy the following properties: taking sufficiently large $n$, we have, for any $x_{i} \in \Gamma_{n}$,

(i) $C_{1} \leq \sharp\left\{x_{j} \in \Gamma_{n} ; x_{i} \sim x_{j}\right\} \leq C_{2}$,

(ii) $n^{d} \operatorname{vol} B\left(x_{i}, \frac{1}{n}\right) \leq C_{3}$,

(iii) $C_{4} \leq n^{d}$ vol $B\left(x_{i}, \frac{1}{3 n}\right)$,

(iv) $C_{5} \leq n^{d}$ vol $B\left(x_{i}, \frac{1}{2 n}\right) \leq C_{6}$,

(v) $\operatorname{vol} B\left(x_{i}, \frac{1}{n}\right) \leq C_{7} \operatorname{vol} B\left(x_{i}, \frac{1}{2 n}\right)$,

(vi) $\sharp\left(\Gamma_{n}\right) \leq C_{8} n^{d}$ vol $M$.

We will show $C=\max \left\{2 C_{2}^{2} C_{3} / C_{4}, 18 C_{2} C_{3} / C_{1} C_{5}\right\}$ is the required constant in the Theorem. We explain how to choose $C_{1}, \ldots, C_{8}$ with the emphasis especially on their independence on the curvature, since this is the reason why we do not need any assumption on the curvatures of $M$ in this paper.

Let $B_{\mathbf{R}^{d}}(r)$ be the ball of radius $r$ in $\mathbf{R}^{d}$, where $d$ is the dimension of $M$, and vol $B_{\mathbf{R}^{d}}(r)$ denote its volume in $\mathbf{R}^{d}$. Then

$$
\lim _{r \rightarrow 0} \frac{\operatorname{vol} B\left(x_{i}, r\right)}{\operatorname{vol} B_{\mathbf{R}^{d}}(r)}=1,
$$

for any $x_{i} \in M$. We call this the limit formula. In the limit formula, the rate of convergence depends on the curvature at $x_{i}$, however, the convergence is uniform since $M$ is compact. 
(i) Since $V\left(\Gamma_{n}\right)$ is $\frac{1}{n}$-separated, $\left\{B\left(x_{i}, \frac{1}{2 n}\right)\right\}_{x_{i} \in \Gamma_{n}}$ are disjoint and if $x_{i} \sim x_{j}$, then $B\left(x_{j}, \frac{1}{2 n}\right) \subset B\left(x_{i}, \frac{3}{n}+\frac{1}{2 n}\right)$. Thus for any $x_{i} \in \Gamma_{n}$,

$$
\sum_{x_{i} \sim x_{j}} \operatorname{vol} B\left(x_{j}, \frac{1}{2 n}\right) \leq \operatorname{vol} B\left(x_{i}, \frac{7}{2 n}\right) .
$$

From the limit formula, we can take $C_{2}=\operatorname{vol} B_{\mathbf{R}^{d}}\left(\frac{7}{2 n}\right) / \operatorname{vol} B_{\mathbf{R}^{d}}\left(\frac{1}{2 n}\right)+1=$ $7^{d}+1$. From the construction of $\frac{1}{n}$-nets, $B\left(x_{i}, \frac{2}{n}\right) \subset \bigcup_{x_{i} \sim x_{j}} B\left(x_{j}, \frac{1}{n}\right)$, for any $x_{i} \in \Gamma_{n}$. Therefore, $\operatorname{vol} B\left(x_{i}, \frac{2}{n}\right) \leq \sum_{x_{i} \sim x_{j}} B\left(x_{j}, \frac{1}{n}\right)$. We can take $C_{1}=$ $\operatorname{vol} B_{\mathbf{R}^{d}}\left(\frac{2}{n}\right) / \operatorname{vol} B_{\mathbf{R}^{d}}\left(\frac{1}{n}\right)-1=2^{d}-1$ by the limit formula.

(ii)-(iv) From the limit formula, the existence of $C_{3}, \ldots, C_{6}$ of the required properties is clear. Using the obvious inequalities

$$
(\sqrt{2} r)^{d} \leq \operatorname{vol} B_{\mathbf{R}^{d}}(r) \leq(2 r)^{d},
$$

we can take $C_{3}=2^{d}, C_{4}=(\sqrt{2} / 3)^{d}, C_{5}=(\sqrt{2} / 2)^{d}, C_{6}=1$.

(v) Take $C_{7}=2^{d}+1$.

(vi) Since $\left\{B\left(x_{i}, \frac{1}{2 n}\right)\right\}_{x_{i} \in \Gamma_{n}}$ are disjoint in $M, \sum_{x_{i} \in \Gamma_{n}} \operatorname{vol} B\left(x_{i}, \frac{1}{2 n}\right) \leq \operatorname{vol} M$. Since $C_{5} / n^{d} \leq \operatorname{vol}\left(x_{i}, \frac{1}{2 n}\right)$ for any $x_{i} \in \Gamma_{n}, \# \Gamma_{n} C_{5} / n^{d} \leq \operatorname{vol} M$. Take $C_{8}=1 / C_{5}=(\sqrt{2})^{d}$.

As stated in the introduction, our result does not need any curvature assumptions on $M$, but the rate of convergence in the Theorem depends on the curvature since the rate of convergence of the limit formula depends on the curvature at $x_{i}$.

Proof of the Theorem. Fix $n$ and denote $\left\{x_{j}\right\}_{j=1}^{\sharp V\left(\Gamma_{n}\right)}=V\left(\Gamma_{n}\right)$. Take a partition of unity $\left\{u_{n, j}\right\}_{j}$ on $M$ with the following properties:

$$
\begin{aligned}
& \text { (i) } \operatorname{supp}\left(u_{n, j}\right) \subset B\left(x_{j}, \frac{2}{n}\right) \text { for each } j, \\
& \text { (ii) } u_{n, j}=1 \text { on } B\left(x_{j}, \frac{1}{3 n}\right), \\
& \text { (iii) }\left(d u_{n, j}(x), d u_{n, j}(x)\right) \leq n^{2}, \text { for any } x \in M .
\end{aligned}
$$

Since $\sum_{j} u_{n, j}=1$,

$$
\sum_{j} d u_{n, j}=0 .
$$

For $x \in M$, if $d\left(x, x_{j}\right)>\frac{2}{n}$, then

$$
d u_{n, j}(x)=0 .
$$

We define a linear operator for each $n$,

$$
S_{n}: L^{2}\left(\Gamma_{n}\right) \rightarrow C^{\infty}(M)
$$

by

$$
S_{n}(f)(x)=\sum_{x_{j} \in \Gamma_{n}} f\left(x_{j}\right) u_{n, j}(x)
$$

for $f \in L^{2}\left(\Gamma_{n}\right)$. From the definition of $S_{n}, S_{n}$ is injective. Thus, for any linear subspace $\mathscr{F}$ in $L^{2}\left(\Gamma_{n}\right)$, we have $\operatorname{dim} \mathscr{F}=\operatorname{dim} S_{n}(\mathscr{F})$. 
Claim 1. Taking sufficiently large $n$,

$$
\left(d S_{n}(f), d S_{n}(f)\right)_{M} \leq \frac{2 C_{2} C_{3}}{n^{d-1}}(d f, d f)_{\Gamma_{n}}
$$

for any $f \in L^{2}\left(\Gamma_{n}\right)$.

Proof of Claim 1. For each $x \in M$, take $x_{k} \in \Gamma_{n}$ with $d\left(x, x_{k}\right) \leq \frac{1}{n}$, and fix it. Then

$$
\begin{aligned}
d S_{n}(f)(x)= & \sum_{x_{j} \in \Gamma_{n}} f\left(x_{j}\right) d u_{n, j}(x) \\
= & \sum_{j}\left(f\left(x_{j}\right)-f\left(x_{k}\right)\right) d u_{n, j}(x) \\
& +f\left(x_{k}\right) \sum_{j} d u_{n, j}(x), \quad \text { by using (1), } \\
= & \sum_{j}\left(f\left(x_{j}\right)-f\left(x_{k}\right)\right) d u_{n, j}(x), \quad \text { by using }(2), \\
= & \sum_{x_{j} \in \Gamma_{n} ; d\left(x, x_{j}\right) \leq \frac{2}{n}}\left(f\left(x_{j}\right)-f\left(x_{k}\right)\right) d u_{n, j}(x) .
\end{aligned}
$$

Since $d\left(x, x_{j}\right) \leq \frac{2}{n}$ and $d\left(x, x_{k}\right) \leq \frac{1}{n}$ imply $d\left(x_{j}, x_{k}\right) \leq \frac{3}{n}$,

$$
\begin{aligned}
\left|d S_{n}(f)(x)\right| & \leq \sum_{x_{j} ; d\left(x_{j}, x_{k}\right) \leq \frac{3}{n}}\left|f\left(x_{j}\right)-f\left(x_{k}\right)\right|\left|d u_{n, j}(x)\right| \\
& \leq \sum_{x_{j} ; x_{j} \sim x_{k}}\left|f\left(x_{j}\right)-f\left(x_{k}\right)\right| n .
\end{aligned}
$$

Thus,

$$
\begin{aligned}
\left(d S_{n}(f)(x), d S_{n}(f)(x)\right) & \leq n^{2}\left(\sum_{x_{j} ; x_{j} \sim x_{k}}\left|f\left(x_{j}\right)-f\left(x_{k}\right)\right|\right)^{2} \\
& \leq n^{2} C_{2} \sum_{x_{j} ; x_{j} \sim x_{k}}\left(f\left(x_{j}\right)-f\left(x_{k}\right)\right)^{2} .
\end{aligned}
$$

Therefore,

$$
\begin{aligned}
\left(d S_{n}(f), d S_{n}(f)\right) & \leq n^{2} C_{2} \sum_{x_{k} \in \Gamma_{n}}\left\{\sum_{x_{j} ; x_{j} \sim x_{k}}\left(f\left(x_{j}\right)-f\left(x_{k}\right)\right)^{2} \operatorname{vol} B\left(x_{k}, \frac{1}{n}\right)\right\} \\
& \leq C_{2} C_{3} \frac{1}{n^{d-1}} \sum_{x_{k} \in \Gamma_{n} x_{j} ; x_{j} \sim x_{k}} \frac{\left(f\left(x_{i}\right)-f\left(x_{k}\right)\right)^{2}}{n} \\
& =\frac{2 C_{2} C_{3}}{n^{d-1}}(d f, d f)_{\Gamma_{n}} .
\end{aligned}
$$

Claim 2. For sufficiently large $n$, we have

$$
\left(S_{n}(f), S_{n}(f)\right)_{M} \geq \frac{C_{4}}{C_{2} n^{d-1}}(f, f)_{\Gamma_{n}}
$$

for any $f \in L^{2}\left(\Gamma_{n}\right)$. 
Proof of Claim 2.

$$
\begin{aligned}
(f, f)_{\Gamma_{n}} & =\sum_{x_{j} \in \Gamma_{n}} f^{2}\left(x_{j}\right) m_{l_{n}}\left(x_{j}\right) \leq \frac{C_{2}}{n} \sum_{x_{j} \in \Gamma_{n}} f^{2}\left(x_{j}\right) \\
& \leq \frac{C_{2}}{n} \frac{n^{d}}{C_{4}} \sum_{x_{j} \in \Gamma_{n}} f^{2}\left(x_{j}\right) \operatorname{vol} B\left(x_{j}, \frac{1}{3 n}\right) \\
& \leq \frac{C_{2}}{C_{4}} n^{d-1} \int_{M}\left(S_{n}(f), S_{n}(f)\right) d V=\frac{C_{2}}{C_{4}} n^{d-1}\left(S_{n}(f), S_{n}(f)\right)_{M} .
\end{aligned}
$$

From Claim 1 and Claim 2 we have the next claim.

Claim 3. For sufficiently large $n$, we have

$$
\frac{\left(d S_{n}(f), d S_{n}(f)\right)_{M}}{\left(S_{n}(f), S_{n}(f)\right)_{M}} \leq \frac{2 C_{2}^{2} C_{3}}{C_{4}} \frac{(d f, d f)_{\Gamma_{n}}}{(f, f)_{\Gamma_{n}}}
$$

for any $f \in L^{2}\left(\Gamma_{n}\right)$.

Using Claim 3, we can show $\lambda_{k}(M) \leq \frac{2 C_{2}^{2} C_{3}}{C_{4}} \liminf _{n} \lambda_{k}\left(\Gamma_{n}, l_{n}\right)$ as follows. From the Lemma, for any $\varepsilon>0$, we can take a $(k+1)$-dimensional linear subspace $\mathscr{F}$ of $L^{2}\left(\Gamma_{n}\right)$ such that

$$
\sup _{f \in \mathscr{F}} \frac{(d f, d f)}{(f, f)} \leq \lambda_{k}\left(\Gamma_{n}\right)+\varepsilon .
$$

From Claim 3, for sufficiently large $n$, we have

$$
\sup _{g \in S_{n}(\mathscr{F})} \frac{(d g, d g)}{(g, g)} \leq \frac{2 C_{2}^{2} C_{3}}{C_{4}} \sup _{f \in \mathscr{F}} \frac{(d f, d f)}{(f, f)} .
$$

Since $\operatorname{dim}(\mathscr{F})=\operatorname{dim}\left(S_{n}(\mathscr{F})\right)=k+1$, we have

$$
\lambda_{k}(M) \leq \sup _{g \in S_{n}(\mathscr{F})} \frac{(d g, d g)}{(g, g)},
$$

from the Lemma. Combining (3)-(5), we have

$$
\lambda_{k}(M) \leq \frac{2 C_{2}^{2} C_{3}}{C_{4}}\left(\lambda_{k}\left(\Gamma_{n}\right)+\varepsilon\right),
$$

for sufficiently large $n$. Since $\varepsilon$ was arbitrary, we thus showed

$$
\lambda_{k}(M) \leq \frac{2 C_{2}^{2} C_{3}}{C_{4}} \liminf _{n \rightarrow \infty} \lambda_{k}\left(\Gamma_{n}, l_{n}\right) .
$$

Next, we define a linear operator for each $n$

$$
T_{n}: C^{\infty}(M) \rightarrow L^{2}\left(\Gamma_{n}\right)
$$

by

$$
T_{n}(f)\left(x_{i}\right)=\frac{\int_{B\left(x_{i}, \frac{1}{n}\right)} f d V}{\operatorname{vol} B\left(x_{i}, \frac{1}{n}\right)},
$$

for $f \in C^{\infty}(M)$ and each $x_{i} \in \Gamma_{n}$. 
This direction, i.e., $C^{\infty}(M) \rightarrow L^{2}\left(\Gamma_{n}\right)$, needs more subtle treatment than the other direction. The reason is, technically, the linear operator $T_{n}$ may decrease the dimension of a subspace of $C^{\infty}(M)$, or philosophically, we have to lose something in this procedure because $T_{n}$ is an approximation of an infinitedimensional space $C^{\infty}(M)$ by a finite-dimensional space $L^{2}\left(\Gamma_{n}\right)$. Therefore, we have to deal with the error terms, such as $\varepsilon C_{7} \mathrm{vol} M$ in Claim 4, etc., in the following argument.

Let $\mathscr{F}$ be a finite-dimensional linear subspace of $C^{\infty}(M)$. We defined $\mathscr{F}(1)$ to denote the set $\{f \in \mathscr{F} \mid(f, f)=1\}$. Then for any $\varepsilon>0$, taking sufficiently large $n$, we have

\section{Claim 4.}

$$
(f, f)_{M} \leq \frac{2 C_{3}}{C_{1} n^{d-1}}\left(T_{n}(f), T_{n}(f)\right)_{\Gamma_{n}}+\varepsilon C_{7} \operatorname{vol} M, \quad \text { for any } f \in \mathscr{F}(1) .
$$

Proof of Claim 4. Taking $n$ sufficiently large, we have

$$
\int_{B\left(x_{i}, \frac{1}{n}\right)}(f, f) d V \leq\left\{2\left(T_{n}(f)\left(x_{i}\right)\right)^{2}+\varepsilon\right\} \operatorname{vol} B\left(x_{i}, \frac{1}{n}\right),
$$

for any $x_{i} \in \Gamma_{n}$ and $f \in \mathscr{F}(1)$ since $\mathscr{F}$ is finite dimensional. Therefore,

$$
\begin{aligned}
(f, f)_{M} & \leq \sum_{i} \int_{B\left(x_{i}, \frac{1}{n}\right)}(f, f) d V \\
& \leq 2 \sum_{i}\left(T_{n}(f)\left(x_{i}\right)\right)^{2} \operatorname{vol} B\left(x_{i}, \frac{1}{n}\right)+\varepsilon \sum_{i} \operatorname{vol} B\left(x_{i}, \frac{1}{n}\right) \\
& \leq \frac{2 C_{3}}{n^{d}} \sum_{i}\left(T_{n}(f)\left(x_{i}\right)\right)^{2}+\varepsilon C_{7} \sum_{i} \operatorname{vol} B\left(x_{i}, \frac{1}{2 n}\right) \\
& \leq \frac{2 C_{3}}{C_{1} n^{d-1}} \sum_{i}\left(T_{n}(f)\left(x_{i}\right)\right)^{2} m_{l_{n}}\left(x_{i}\right)+\varepsilon C_{7} \operatorname{vol} M \\
& =\frac{2 C_{3}}{C_{1} n^{d-1}}\left(T_{n}(f), T_{n}(f)\right)_{\Gamma_{n}}+\varepsilon C_{7} \operatorname{vol} M .
\end{aligned}
$$

Also, for any $\varepsilon>0$, taking sufficiently large $n$, we have

\section{Claim 5.}

$$
\begin{aligned}
\left(d T_{n}(f),\right. & \left.d T_{n}(f)\right)_{\Gamma_{n}} \\
\leq & n^{d-1}\left\{\frac{9 C_{2}}{C_{5}}(d f, d f)_{M}+\varepsilon \frac{9 C_{2}}{2 C_{5}} \text { vol } M\right\} \text { for any } f \in \mathscr{F}(1) .
\end{aligned}
$$

Proof of Claim 5. Since $\mathscr{F}$ is finite dimensional, taking $n$ sufficiently large, we have, for any $x_{i}, x_{j} \in \Gamma_{n}$ with $x_{i} \sim x_{j}$,

$$
\left(T_{n}(f)\left(x_{i}\right)-T_{n}(f)\left(x_{j}\right)\right)^{2} \leq\left\{\frac{2 \int_{B\left(x_{i}, \frac{1}{2 n}\right)}(d f, d f) d V}{\operatorname{vol} B\left(x_{i}, \frac{1}{2 n}\right)}+\varepsilon\right\} d^{2}\left(x_{i}, x_{j}\right),
$$


and since $d^{2}\left(x_{i}, x_{j}\right) \leq \frac{9}{n^{2}}$,

$$
\leq \frac{18}{n^{2}} \frac{n^{d}}{C_{5}} \int_{B\left(x_{i}, \frac{1}{2 n}\right)}(d f, d f) d V+\frac{9}{n^{2}} \varepsilon
$$

Therefore,

$$
\begin{aligned}
\left(d T_{n}(f), d T_{n}(f)\right)_{\Gamma_{n}} & =\frac{1}{2} \sum_{x_{i} \sim x_{j}}\left(T_{n}(f)\left(x_{i}\right)-T_{n}(f)\left(x_{j}\right)\right)^{2} n \\
& \leq \frac{9 C_{2} n^{d-1}}{C_{5}} \sum_{x_{i} \in \Gamma_{i}} \int_{B\left(x_{i}, \frac{1}{2 n}\right)}(d f, d f) d V+\varepsilon \frac{9 C_{2}}{2 n} \sharp\left(\Gamma_{n}\right) \\
& \leq \frac{9 C_{2} n^{d-1}}{C_{5}} \int_{M}(d f, d f) d V+\varepsilon \frac{9 C_{2} n^{d-1}}{2 C_{5}} \operatorname{vol} M \\
& =\frac{9 C_{2} n^{d-1}}{C_{5}}(d f, d f)_{M}+\varepsilon \frac{9 C_{2} n^{d-1}}{2 C_{5}} \operatorname{vol} M .
\end{aligned}
$$

Combining Claim 4 and Claim 5, we have the next claim.

Claim 6. Let $\mathscr{F}$ be a finite-dimensional linear subspace of $C^{\infty}(M)$. Then for any small $\varepsilon>0$, taking sufficiently large $n$, we have

$$
\frac{\left(d T_{n}(f), d T_{n}(f)\right)_{\Gamma_{n}}}{\left(T_{n}(f), T_{n}(f)\right)_{\Gamma_{n}}} \leq \frac{18 C_{2} C_{3}}{C_{1} C_{5}} \frac{(d f, d f)_{M}+\varepsilon}{(f, f)_{M}-\varepsilon}
$$

for any $f \in \mathscr{F}(1)$.

Using Claim 6, for any $\varepsilon>0$, taking sufficiently large $n$, we can show that

$$
\lambda_{k}\left(\Gamma_{n}, l_{n}\right) \leq \frac{18 C_{2} C_{3}}{C_{1} C_{5}}\left(\lambda_{k}(M)+\varepsilon\right)
$$

as we showed (6) from Claim 3. The argument is similar, except that the linear operator $T_{n}$ may decrease the dimension of a subspace of $C^{\infty}(M)$, however, for any finite-dimensional subspace $\mathscr{F}$ of $C^{\infty}(M)$ and any $\varepsilon>0$, we can take a subspace $\mathscr{F}^{\prime}$ by slightly perturbing $\mathscr{F}$ in $C^{\infty}(M)$ such that $\operatorname{dim} \mathscr{F}=\operatorname{dim} \mathscr{F}^{\prime}=\operatorname{dim} T_{n}\left(\mathscr{F}^{\prime}\right)$ and

$$
\sup _{f \in \mathscr{F}^{\prime}} \frac{(d f, d f)}{(f, f)} \leq \sup _{f \in \mathscr{F}} \frac{(d f, d f)}{(f, f)}+\varepsilon
$$

Filling out the details is left to the reader. From (8), we have

$$
\limsup _{n \rightarrow \infty} \lambda_{k}\left(\Gamma_{n}, l_{n}\right) \leq \frac{18 C_{2} C_{3}}{C_{1} C_{5}} \lambda_{k}(M) .
$$

Therefore, taking $C=\max \left\{2 C_{2}^{2} C_{3} / C_{4}, 18 C_{2} C_{3} / C_{1} C_{5}\right\}$, we have the Theorem from (7) and (9). It is easy to check $C \leq 2 \cdot 50^{d}$ from the values of $C_{1}, \ldots, C_{8}$ explicitly given before.

\section{REFERENCES}

[B] B. Brooks, The spectral geometry of k-regular graphs, J. Analyse Math. 57 (1991), 120-151.

[C] I. Chavel, Eigenvalues in Riemannian geometry, Academic Press, New York, 1984. 
[D] J. Dodziuk, Finite difference approach to the Hodge theory of Harmonic forms, Amer. J. Math. 98 (1976), 79-104.

[F] K. Fujiwara, On the bottom of the spectrum of the Laplacian on graphs, Geometry and Its Applications (T. Nagano et al., eds.), World Scientific, Singapore, 1993, pp. 21-27.

[K] M. Kanai, Analytic inequalities, and rough isometries between non-compact Riemannian manifolds, Curvature and Topology of Riemannian Manifolds (K. Shiohama et al., eds.), Lecture Notes in Math., vol. 1201, Springer, New York, 1986, pp. 122-137.

Department of Mathematics, Keio University, Yokohama 223, Japan

E-mail address: fujiwara@math.keio.ac.jp 